Rev. High Pressure Sci. Technol., Vol. 7 (1998) 1177 1179

\title{
Measurement of Ultrasound Velocity in Condensed Helium and Neon Gases
}

\author{
Y. Hanayama, M. Kimura, and T. Nishitake* \\ Faculty of Engineering, Ehime University, Matsuyama, Ehime 790-77, Japan \\ *Faculty of Management and Information Science, Shikoku University, Tokushima, Tokushima 771-11, Japan
}

\begin{abstract}
The ultrasound velocities in condensed helium and neon gases have been measured up to 3.0 and $3.7 \mathrm{GPa}$ at 295 $\mathrm{K}$, respectively. The velocity was measured with high accuracy by a pulse echo method and using lithium niobate single crystal as the ultrasound transducer. Behaviors of condensed state of monoatomic gases have been discussed from compressibilities and heat capacity ratio.

[ sound velocity, helium, neon, compressibility, high pressure ]
\end{abstract}

\section{Introduction}

The knowledge of condensed state of fluid are invaluable in developing model of molecule and testing intermolecular potential. Recently, properties of gases were studied by using many type of intermolecular potential function [1,2].

The ultrasound velocity is very useful for studying their physical properties under high pressures. Experimentally, many authors measured sound velocity in helium[3-8] and neon[5,6,9-14] gases under high pressures. Recently, Mills et al.[7] measured simultaneously the pressure, volume, temperature, and sound velocity in helium to $2 \mathrm{GPa}$ and calculated equation of state. Kortbeek et al. measured the density and sound velocity in helium[8] and neon[14] to $1 \mathrm{GPa}$ and calculated the thermodynamic properties up to 6 $\mathrm{GPa}$ from equation of state based on statistical mechanical perturbation theory [15] .

In this work, the ultrasound velocities in helium and neon have been measured above GPa region and at $295 \mathrm{~K}$. Compressibilities and heat capacity ratio are calculated and the behaviors of high pressure condensed state of gases are discussed.

\section{Compressibilities of gases}

The velocity of sound in gas is connected with the thermodynamic functions, such as the isothermal compressibility $\chi_{T}$ and the adiabatic one $\chi_{S}$ are defined by the following identities:

$$
\chi_{T}=-\frac{1}{V}\left(\frac{\partial V}{\partial p}\right)_{T}=\frac{1}{\rho}\left(\frac{\partial \rho}{\partial p}\right)_{T}
$$

and

$$
\chi_{S}=-\frac{1}{V}\left(\frac{\partial V}{\partial p}\right)_{S}=\frac{1}{\rho u^{2}},
$$

where $V$ is the volume, $p$ the pressure, $\rho$ the density and $u$ the ultrasound velocity, respectively. The two compressibilities are related by

$$
\gamma=\frac{C_{p}}{C_{V}}=\frac{\chi_{T}}{\chi_{S}}=u^{2}\left(\frac{\partial \rho}{\partial p}\right)_{T},
$$

where $\gamma$ is the heat capacity ratio, and $C_{p}$ and $C_{V}$ are the heat capacities at constant pressure and volume, respectively.

\section{Experimental \\ High pressure experiment}

The high pressure apparatus of piston-cylinder type was described in our previous paper[16]. High pressure experiments were done as following. Gas of more than 99.99 percent purity is compressed with a preliminary gas system up to $370 \mathrm{MPa}$. Then gas is introduced to the high pressure cylinder and the gas pressure is raised in increment. After equilibration at room temperature, the ultrasound velocity and the piston displacement are measured. The piston displacement can be measured by two dial gauges fitted with the lower and upper rams.

\section{High pressure measurements}

For a sound velocity measurement, a detail of the sound device was also described in the above mentioned paper[16]. In this study, lithium niobate $\left(\mathrm{LiNbO}_{3}\right)$ single crystal of $36^{\circ} \mathrm{Y}$-cut having the fundamental frequency of $6.6 \mathrm{MHz}$ was employed as ultrasound transducer substituted the ceramic one made by lead zirconate titanate. These crystals as emitter and receiver are placed at distance of 20 $\mathrm{mm}$ to act and fitted on spacer with spring to parallel each other.

The ultrasound waves propagate a distance of spacer length $L$, after transit time arrive at receiver, and are observed as primary pulse. After the arrived waves are reflected at receiver and at emitter, they are again observed at receiver as the 2 nd echo pulse. The ultrasound velocity $u$ is obtained in the following equation:

$$
u=\frac{2 L}{T}
$$

where $T$ is the duration time between 1 st and 2 nd pulse, and is measured with a high accuracy by the oscilloscope's delay time technique.

Pressure of gas is obtained from electric resistance of manganin wire, located inside the cylinder. The gauge was calibrated with the freezing point of mercury. 


\section{Experimental results}

The ultrasound velocities in helium gas have been measured up to $3.01 \mathrm{GPa}$ at $295 \mathrm{~K}$. The same ones in neon gas have been measured up to $3.71 \mathrm{GPa}$ at $295 \mathrm{~K}$. This accuracy of measurement is less than $0.05 \%$. Its standard deviation is $1.0 \mathrm{~m} / \mathrm{s}$.

\section{Sound velocity in helium}

The experimental results on velocity in helium are shown in Fig.1. The velocity increases smoothly with pressure up to maximum pressure, and the rate of increase shows slowing down.

The velocities are in good agreement within $1.0 \%$ for the measured ones by Kortbeek et al.[8] up to $1 \mathrm{GPa}$. Above 1 $\mathrm{GPa}$, the present results of velocity are in agreement within 0.3 and $0.2 \%$ at 1 and $2 \mathrm{GPa}$ with the calculated values from equations given by Mills et al.[7], respectively. For early our data up to $1.5 \mathrm{GPa}$ measured by a single pulse method with frequency of $2 \mathrm{MHz}$ lead zirconate titanate transducer, the present values obtained by lithium niobate one are maximum $1.6 \%$ higher.

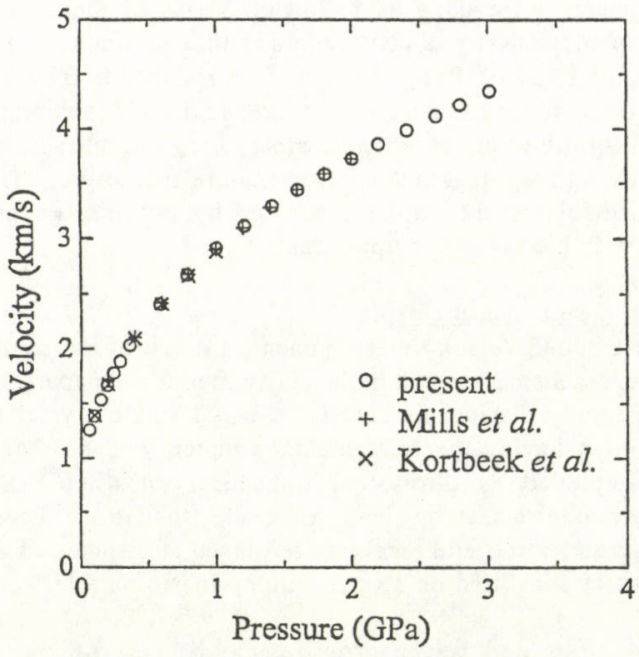

Fig.1. Experimental results on the velocity of sound in helium as a function of pressure at $295 \mathrm{~K}$.

\section{Sound velocity in neon}

In Fig.2, the ultrasound velocities in neon are shown. The velocity increases smoothly with pressure up to 3.71 $\mathrm{GPa}$, and the rate of increase shows slowing down.

The present values of velocity agree with measurement by Pitaevskaya et al. [10] up to $400 \mathrm{MPa}$ at $298.15 \mathrm{~K}$ with an estimated error of $0.3 \%$. Vidal et al. $[5,6]$ measured ultrasound velocity up to $1 \mathrm{GPa}$ at frequency of $15 \mathrm{MHz}$ and Kortbeek et al.[14] measured ultrasound velocity up to 1 $\mathrm{GPa}$ and at frequency of $9.5-10.5 \mathrm{MHz}$. The present results are in agreement within $1.1 \%$ with Vidal and $0.9 \%$ with Kortbeek, respectively.
Above $1 \mathrm{GPa}$, the data are in good agreement within from $0.15 \%$ to $0.7 \%$ with the estimated ones by Kortbeek et al. [15].

Previously, we measured velocity in neon up to $3.4 \mathrm{GPa}$ by old methods using the transducer of lead zirconate titanate. The present values obtained by lithium niobate are maximum $4.5 \%$ higher than the former.

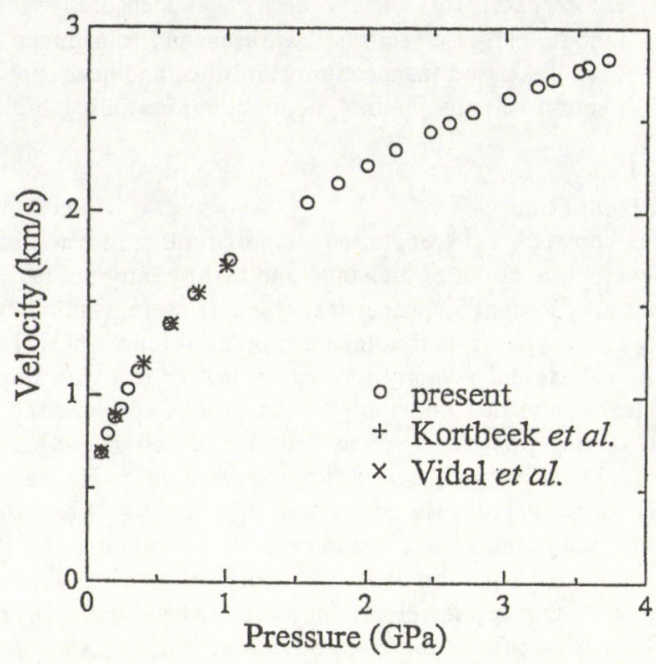

Fig.2. Experimental results on the velocity of sound in neon as a function of pressure at $295 \mathrm{~K}$.

\section{Discussions}

\section{Compressibilities}

The isothermal and adiabatic compressibilities are calculated from eqs.(1) and (2), using both the velocity and density data. In calculations, the velocity ones are employed the present results, but the density ones are referred in the values described by other authors [7,15].

For ideal gas, the isothermal compressibility varies inversely as pressure. Therefore, the product of pressure and compressibility $p \cdot \chi$ is constant and unity. In this paper, compressibilities have been examined as the product value.

For helium, the density data were used ones computed by Mills et al.[7]. The values of $p \cdot \chi_{T}$ and $p \cdot \chi_{S}$ decrease monotonously with increasing pressure, these rates of decrease go down gradually as shown in Fig.3. Both $p \cdot \chi_{T}$ and $p \cdot \chi_{S}$ nearly approach to the values of 0.34 and 0.27 at $3 \mathrm{GPa}$, respectively.

For neon, the density data were used ones computed by Kortbeek et al.[15]. Both the product values decrease monotonously with increasing pressure, these rates of decrease go down gradually as shown in Fig.4. Both $p$. $\chi_{T}$ and $p \cdot \chi_{S}$ nearly approach to the values of 0.27 and 0.21 at $3.7 \mathrm{GPa}$, respectively. 


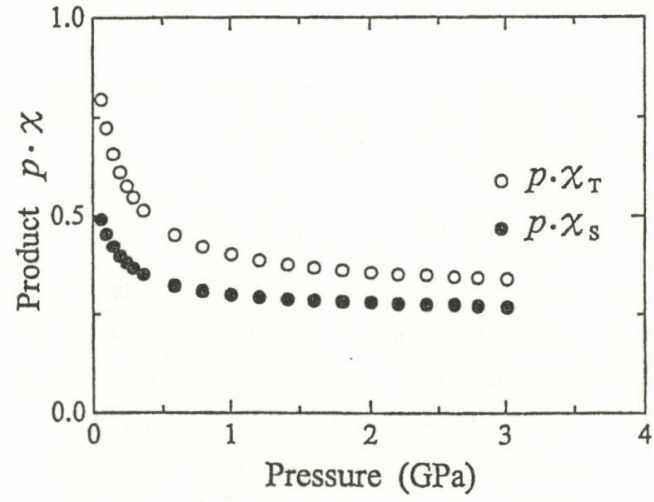

Fig.3. The product of pressure and compressibility $p \cdot \chi$ of helium as a function of pressure at $295 \mathrm{~K}$.

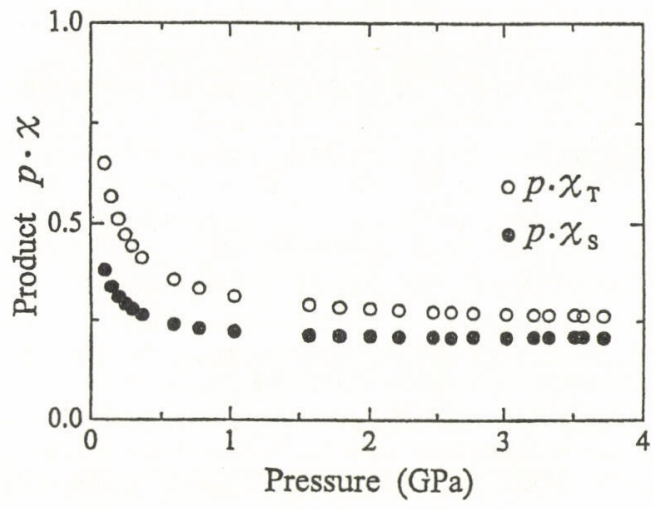

Fig.4. The product of pressure and compressibility $p \cdot \chi$ of neon as a function of pressure at $295 \mathrm{~K}$.

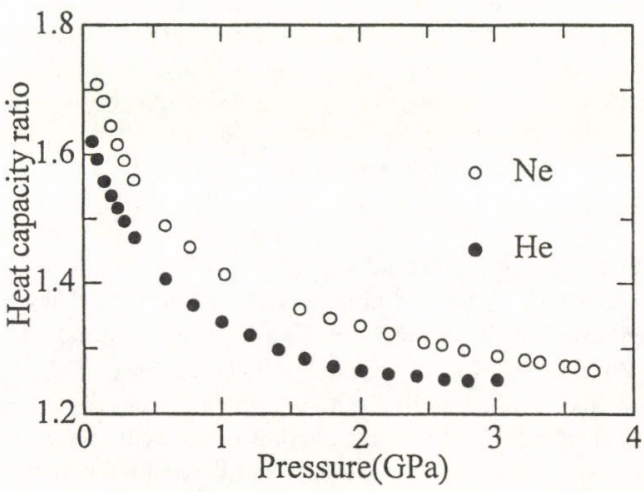

Fig.5. Heat capacity ratios of helium and neon as a function of pressure at $295 \mathrm{~K}$.
Heat capacity ratio under pressure

The heat capacity ratio $\gamma$ of gases are calculated by eq.(3), using both the compressibilities. The $\gamma$ decrease monotonously with increasing pressure, these rates of decrease go down gradually in both gases as shown in Fig.5.

The $\gamma$ of helium and neon are 1.25 at $3.01 \mathrm{GPa}$ and 1.26 at $3.71 \mathrm{GPa}$, respectively. Both the values are smaller than the value of triatomic gas 1.29. These may indicate extreme clustering in the highly compressed fluid.

\section{Conclusions}

The ultrasound velocities have been measured in helium up to $3.01 \mathrm{GPa}$, in neon up to $3.71 \mathrm{GPa}$ at $295 \mathrm{~K}$. The values of product of compressibility and pressure approach to constant under high pressure. Heat capacity ratios of both dense helium and neon decrease monotonously approaching the value of triatomic gas.

\section{Acknowledgments}

The authors would like to express their sincere thanks to many members of Ehime University High Pressure Group for generous assistance in carrying out these experiments.

\section{References}

[1] W.L.Vos, J.A.Schouten, D.A.Young, and M.Ross, J. Chem.Phys., 94, 3835(1991).

[2] R.Le Toullec, P.Loubeyre, and J.P.Pinceaux, Phys. Rev., B40, 2368 (1989).

[3] L.L.Pitaevskaya and A.V.Bilevich, Russ.J.Phys.Chem. 44, 897 (1970).

[4] T.Nishitake and Y.Hanayama, J.Phys.Soc.Jpn., 39, 1065 (1975).

[5] D.Vidal, L.Guengant, and M.Lallemand, Physica, 96A, 545 (1979).

[6] D.Vidal ,R.Tufeu, Y.Garrabos, and B.Le Neindre, High Press. Sci. and Techn., 2, 692(Pergamon Press, Paris, 1980).

[7] R.L.Mills, D.H.Liebenberg, and J.C.Bronson, Phys.Rev., B21, 5137 (1980).

[8] P.J.Kortbeek, J.J. van de Ridder, S.N.Biswas, and J.A. Schouten, Int.J.Thermodynamics, 9, 425(1988).

[9] A.Michels, T.Wassenaar, and G.J.Wolkers, Physica, 31, 237 (1960).

[10] L.L.Pitaevskaya and A.V.Bilevich, High temperaturesHigh pressures, 5, 459 (1973).

[11] V.Ya.Maslennikova, A.N.Egorov, and D.S.Tsiklis, Sov. Phys. Dokl., 21, 440(1976).

[12] P.J.Kortbeek, S.N.Biswas, and J.A.Schouten, Int.J. Thermophys., 9, 803 (1988).

[13] M.Kimura, Y.Hanayama, and T.Nishitake, Jpn.J.Appl. Phys., 26, 1366 (1987).

[14] P.J.Kortbeek and J.A.Schouten, Mol.Phys., 69, 981 (1990).

[15] P.J.Kortbeek, C.A.ten Seldam, and J.A.Schouten, Mol. Phys., 69, 1001 (1990).

[16] M.Kimura, Y.Hanayama, and T.Nishitake, Jpn.J.Appl. Phys., 26, 1361 (1987). 